\title{
Interaction of perfluoroalkyl acids with human liver fatty acid-binding protein
}

\author{
Nan Sheng $\cdot$ Juan Li $\cdot$ Hui Liu $\cdot$ Aiqian Zhang $\cdot$ \\ Jiayin Dai
}

Received: 22 July 2014 / Accepted: 15 October 2014 / Published online: 5 November 2014

(C) Springer-Verlag Berlin Heidelberg 2014

\begin{abstract}
Perfluoroalkyl acids (PFAAs) are highly persistent and bioaccumulative, resulting in their broad distribution in humans and the environment. The liver is an important target for PFAAs, but the mechanisms behind PFAAs interaction with hepatocyte proteins remain poorly understood. We characterized the binding of PFAAs to human liver fatty acid-binding protein (hL-FABP) and identified critical structural features in their interaction. The binding interaction of PFAAs with hL-FABP was determined by fluorescence displacement and isothermal titration calorimetry (ITC) assay. Molecular simulation was conducted to define interactions at the binding sites. ITC measurement revealed that PFOA/PFNA displayed a moderate affinity for hL-FABP at a 1:1 molar ratio, a weak binding affinity for PFHxS and no binding for PFHxA. Moreover, the interaction was mainly mediated by electrostatic attraction and hydrogen bonding. Substitution of Asn111 with Asp caused loss of binding affinity to PFAA, indicating its crucial role for the initial PFAA binding to the outer binding site. Substitution of Arg122 with Gly caused only one molecule of
\end{abstract}

Electronic supplementary material The online version of this article (doi:10.1007/s00204-014-1391-7) contains supplementary material, which is available to authorized users.

N. Sheng $\cdot$ H. Liu $\cdot$ J. Dai $(\bowtie)$

Key Laboratory of Animal Ecology and Conservation

Biology, Institute of Zoology, Chinese Academy of Sciences,

Beijing 100101, People's Republic of China

e-mail: daijy@ioz.ac.cn

J. Li · A. Zhang $(\bowtie)$

State Key Laboratory of Environmental Chemistry

and Ecotoxicology, Research Center for Eco-Environmental

Sciences, Chinese Academy of Sciences, Beijing 100085,

People's Republic of China

e-mail: aqzhang@rees.ac.cn
PFAA to bind to hL-FABP. Molecular simulation showed that substitution of Arg122 increased the volume of the outer binding pocket, making it impossible to form intensive hydrophobic stacking and hydrogen bonds with PFOA, and highlighting its crucial role in the binding process. The binding affinity of PFAAs increased significantly with their carbon number. $\operatorname{Arg} 122$ and Asn111 played a pivotal role in these interactions. Our findings may help understand the distribution pattern, bioaccumulation, elimination, and toxicity of PFAAs in humans.

Keywords Perfluorinated compounds · Human liver fatty acid-binding protein $\cdot$ Interaction $\cdot$ Isothermal titration calorimetry $\cdot$ Molecular simulation

\section{Introduction}

Perfluoroalkyl acids (PFAAs) are a family of perfluoroalkyl and polyfluoroalkyl substances (PFASs) with unique physical and chemical characteristics consisting of a carbon backbone and charged functional moiety like carboxylate, sulfonate, or phosphonate (Lau et al. 2007). Many PFAAs, which are highly resistant to degradation and environmentally persistent due to strong $\mathrm{C}-\mathrm{F}$ bonds (Weiss et al. 2009), have been widely used in commercial and industrial products (Renner 2001). Because of their wide use in recent decades, PFAAs have been detected in the liver, fat, and serum of wildlife (De Silva and Mabury 2006) and in the serum and blood of humans (Kannan et al. 2004). The elimination half-lives of perfluorooctanoic acid (PFOA) and perfluorooctanesulfonate (PFOS) in serum varies widely by species and sex and are considered long (up to 3.8 and 5.4 years) in human blood (Lau et al. 2007). Due to their environmental persistence, bioaccumulation and 
biomagnification through the food web, as well as their presence in humans and wildlife, the potential health risks of PFAAs are of great concern on an international scale.

The toxicity and human health effect of PFAAs have been investigated in the past decade. Exposure to PFAAs $(C>7)$ in laboratory animals was found to increase the liver-to-body weight ratio, lipid levels, and peroxisome proliferation and reduce serum cholesterol and triacylglycerol (Kennedy et al. 2004). PFAAs have been shown to activate the peroxisome proliferator-activated receptor $\alpha(\operatorname{PPAR} \alpha)$ (Kennedy et al. 2004). Once activated, PPAR $\alpha$ can regulate the transcription of genes involved in a number of biological processes, including lipid metabolism, inflammation, and cell growth. In addition, the structural resemblance of PFAAs to natural fatty acids has raised concern that PFAAs may disrupt fatty acid binding with some important transport proteins and disrupt lipid regulation (Lau et al. 2007). Some epidemiological studies have shown positive associations between PFOA and lipids in general human populations. For example, in occupationally exposed employees, increased PFOA was associated with increased total cholesterol and triglycerides (Olsen et al. 2007; Sakr et al. 2007). Recently, a much larger study of adults from environmentally exposed and adjacent communities reported a positive association between PFOA and total and low-density lipoprotein cholesterol, and a less clear association of PFOA with triglycerides (Steenland et al. 2009). As these epidemiological data are inconsistent, adverse effects of PFAAs to humans and the mechanism of action need further exploration.

Previous studies have shown that the liver is an important target for PFAAs. Liver fatty acid-binding protein (L-FABP or FABP1) is the most broadly distributed mammalian FABP and is highly expressed in the liver as well as in the intestine and kidneys. FABPs are members of the intracellular lipid-binding protein superfamily. They play an important role in uptake, sequestering, and transport of long-chain fatty acids and interact with transport and enzyme systems, as well as gene expression regulation (Atshaves et al. 2010; Bernlohr et al. 1997). L-FABP has a high affinity and capacity to bind to long-chain fatty acid and its oxidation products. Previous studies have shown that PFAAs may, with a similar structure to fatty acids, successfully compete and bind with these natural ligands for L-FABP (Luebker et al. 2002; Zhang et al. 2013). Human L-FABP (hL-FABP) may be a potential cytosolic transporter for these major pollutants within the hepatocyte cytoplasm to the nucleus for PPAR-mediated transcriptional regulation and detoxification pathways. However, mechanistic details of PFAAs binding with hL-FABP have not been carefully investigated.

In this study, we characterized the binding of PFAAs to wild-type (WT) hL-FABP and its variants, and identified key residues in interaction with PFAAs. This research will further our understanding about the effect of PFAAs on human livers and how they interact with each other such that their potential risk to humans can be evaluated.

\section{Materials and methods}

\section{Chemicals}

Octanoic acid (OA, purity $\geq 99 \%$ ), perfluorohexanoic acid (PFHxA, $>97 \%$ ), perfluorooctanoic acid (PFOA, >96\%), perfluorononanoic acid (PFNA, $>97 \%$ ), tridecafluorohexane-1-sulfonic acid potassium salt (PFHxS, $>98 \%$ ), thrombin, and 8-anilino-1-naphthalenesulfonic acid (1,8-ANS, $\geq 97 \%$ ) were purchased from Sigma (St. Louis, MO). Sequencing-grade trypsin was purchased from Promega (Madison, WI).

Overexpression and purification of WT hL-FABP and its variants

Full-length WT hL-FABP was cloned into the pET-28a vector between the BamH I and Xho I restriction sites. To obtain recombinant protein consisting of 127 amino acids in accordance with WT hL-FABP, we inserted a $5^{\prime}$ thrombin restriction enzyme cutting site before the target gene and mutated the original thrombin restriction enzyme cutting site (CGC to CCC) in the pET-28a vector. The insert sequence was confirmed by DNA sequencing. The resultant plasmid was transformed to the BL21 (DE3) strain of Escherichia coli. Following IPTG $(1 \mathrm{mM})$ induction at a cell density of 0.6 , recombined protein was overexpressed for $18 \mathrm{~h}$ at $23{ }^{\circ} \mathrm{C}$. Bacterial cells were lysed by sonication in a buffer containing $1 \mathrm{mM}$ PMSF and $1 \mathrm{mM}$ DTT. Affinity purification was performed on a HisTrap HP column on an AKTA FPLC system (GE Healthcare, USA). The details for protein purification are given in the supplementary information. Protein concentration was determined by the bicinchoninic acid method.

The hL-FABP variants (S39G, M74G, N111D, and R122G) were mutated from WT hL-FABP using a Fast Mutagenesis System (TransGen Biotech, China). Overexpression, purification, and identification of proteins were the same as described above.

\section{Circular dichroism spectroscopy}

After incubation with various concentrations of PFAAs, CD spectra of $5 \mu \mathrm{M}$ hL-FABP (WT and its variants) in buffer B were collected on a Chirascan Plus spectrometer (Applied Photophysics, Leatherhead, UK) at room temperature with a 1-mm path length quartz cuvette. The spectra were recorded 
from 193 to $250 \mathrm{~nm}$ with a $0.5 \mathrm{~nm}$ bandwidth, $1-\mathrm{nm}$ increment and 0.5-s collection time per step. Three scans were averaged as the final spectra data for secondary structure analysis. The spectra of buffer B were obtained the same way and were subtracted from the protein spectra. The $\alpha$-helix and $\beta$-sheet contents of proteins were estimated with the CDSSTR algorithm (Kelly et al. 2005; Sreerama and Woody 2000), which gave the smallest value of RMSD.

\section{Fluorescence binding assays}

Fluorescence emission spectra were measured on a Horiba Fluoromax-4 spectrofluorometer (Edison, NJ) in buffer $\mathrm{C}(50 \mathrm{mM}$ Tris- $\mathrm{HCl}, \mathrm{pH} 8.0)$ at room temperature under steady-state conditions. Fluorometric titrations of 1,8-ANS into hL-FABP and PFAAs displacement were performed as described previously (Carbone and Velkov 2013; Velkov et al. 2005), with an adjustment in protein concentration $(0.25 \mu \mathrm{M})$. All data modeling operations were performed as previously described using GraphPad Prism V5.0 (GraphPad software, San Diego, CA, USA). Details are given in the supplementary information.

\section{Isothermal titration calorimetry (ITC)}

The ITC experiments on the binding of PFAAs to WT hLFABP and its variants were carried out by iTC200 titration calorimetry (MicroCal, GE Healthcare) at $25{ }^{\circ} \mathrm{C}$. Freshly purified proteins in buffer B were diluted with the same buffer and loaded into the sample cell $(200 \mu \mathrm{l})$. Solutions of PFAAs in buffer B were placed in an injection syringe $(40 \mu \mathrm{l})$. Concentrations of proteins and PFAAs were changed according to the different proteins or PFAAs (see Table S1). The first PFAA injection was $0.5 \mu \mathrm{l}$, and the following 19 injections were $2 \mu \mathrm{l}$. The stirring rate was $1,000 \mathrm{rpm}$. The time between the titration steps was $120 \mathrm{~s}$ to reach an equilibrium state. Dilution heats of PFAAs were measured by injecting PFAAs solution into buffer alone. The resulting data were fitted to the one site of the site binding model using Microcal ORIGIN Software with subtracting dilution heats of PFAAs beforehand. All ITC experiments were repeated three times to ensure reproducibility.

\section{Limited proteolysis of hL-FABP}

Before the experiment, proteins were changed into buffer D (50 mM Tris-HCl, $2 \mathrm{mM} \mathrm{CaCl}_{2}, 5 \%$ Glycerin, $\mathrm{pH}$ 8.0) with a HiTrap Desalting column. Proteins $(50 \mu \mathrm{M})$ were pre-equilibrated with PFOA $(0,1,5,25$, and $50 \mu \mathrm{M}$ final PFOA concentrations) for $30 \mathrm{~min}$ at $20^{\circ} \mathrm{C}$, and then trypsin was added at a ratio of 20:1 (protein/protease, w/w). Digestion was performed at $37{ }^{\circ} \mathrm{C}$ for $8 \mathrm{~h}$, followed by the addition of $1 \mu 1$ of $50 \mathrm{mM}$ PMSF to stop the digestion. Samples were heated and then resolved on $4 \%$ stacking, $20 \%$ resolving polyacrylamide gels at $4{ }^{\circ} \mathrm{C}$. Gels were stained with Coomassie Blue G-250 and destained with $5 \%$ methanol/10\% acetic acid solution.

Molecular simulations

All molecular simulations were performed on a DELL Precision 370 work station with a SYBYL-X 1.2 software package from Tripos, Inc., Co. (SYBYL-X, version 1.2; Tripos International: St. Louis, MO, 2010). The geometry optimizations of the tested molecules were conducted with the standard Tripos Force Field and the conjugate-gradient minimization with an energy change convergence criterion of $0.05 \mathrm{kcal} / \mathrm{mol}$. Atomic charges were computed using the Gasteiger Huckel charge field. The X-ray crystal structures of hL-FABP (PDB ID: 3STK) were obtained from the Protein Data Bank (PDB, http://www.rcsb.org/pdb). The binding pose of the tested compounds with hL-FABP was subsequently investigated using a flexible docking method with the Tripos Surflex-Dock program. Moreover, further computations were performed on the Surflex-Dock-simulated lowest-energy ligand-protein complex using energy minimization and molecular dynamics along with an AMBER7 FF99 force field to obtain more accurate poses.

\section{Results}

Effect of PFAAs on the secondary structure of hL-FABP by CD spectroscopy

To investigate the interactions of PFAAs with hL-FABP, the solution structure of WT hL-FABP and its variants before and after the addition of different concentrations of PFAAs was examined using circular dichroism. The far-UV CD spectra of the recombinant hL-FABP exhibited a negative minima at $218 \mathrm{~nm}$ and a shoulder at $195 \mathrm{~nm}$ (Fig. 1), indicating typical structures rich in $\beta$-sheet. The recombinant WT hL-FABP contained $17.1 \% \alpha$-helix and $50.3 \% \beta$-sheet (Table 1), similar to the contents of the secondary structure (12.6\% $\alpha$-helix and $59.1 \% \beta$-sheet) predicted by PredictProtein (http://www.predictprotein.org). The $\alpha$-helix and $\beta$-sheet contents of S39G, M74G, and N111D exhibited no significant changes compared to those of wild type, showing that these variants retained secondary structures similar to the WT protein. For R122G; however, the helical content decreased to $15.3 \%$, and its sheet amount increased minimally, indicating that secondary structures were affected due to Arg122 substitution with glycine (Table 1). With the addition of PFAAs, CD spectral change depended on the type of chemicals and substitution. After adding PFHxS and PFHxA, no measurable changes in CD spectra of WT hL-FABP and 

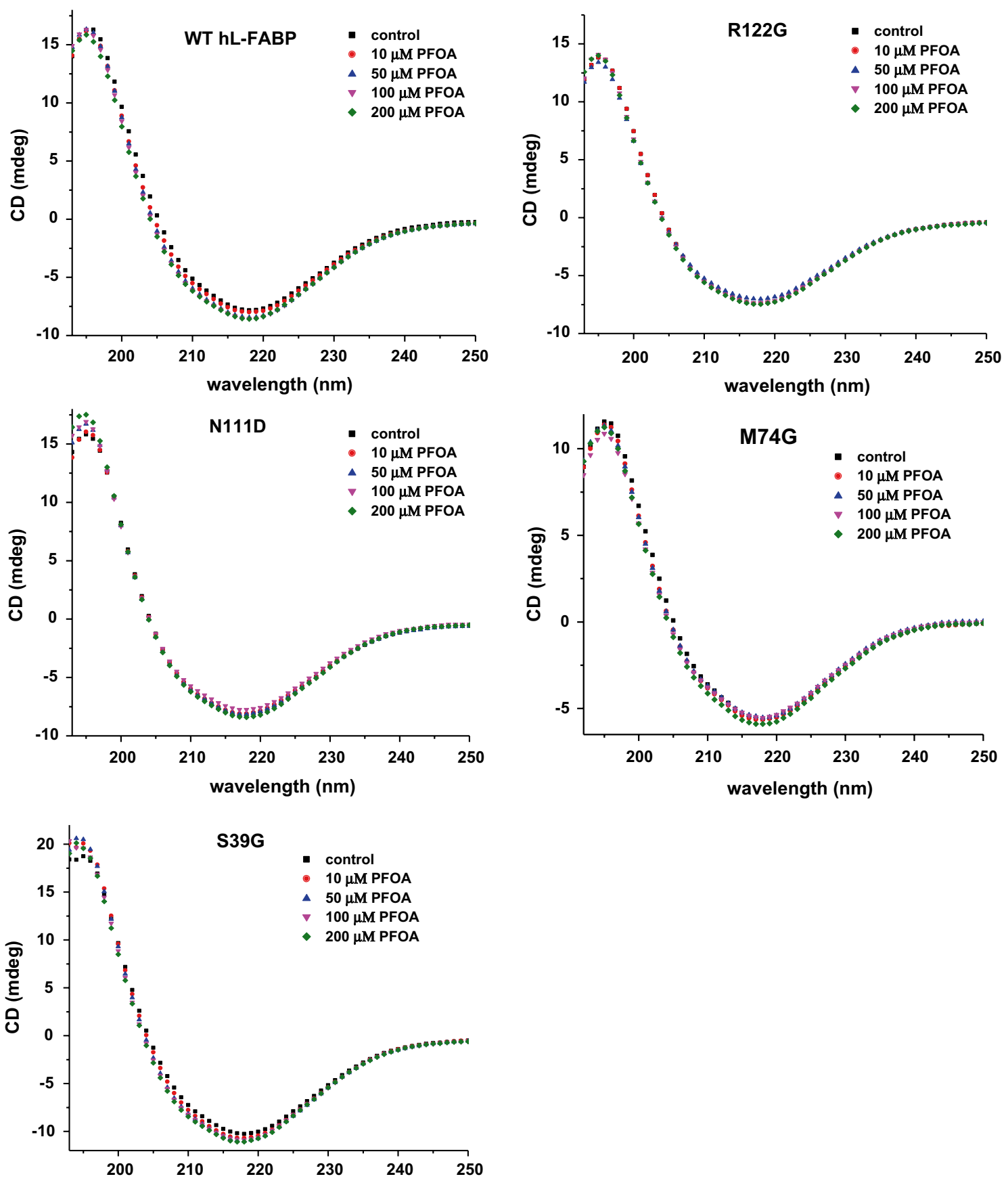

Fig. 1 CD spectra of different concentrations of PFOA added to $5 \mu \mathrm{M} \mathrm{WT}$ hL-FABP and its four variants (S39G, M74G, N111D, and R122G), respectively

its variants were observed (Figure S1, Table S1). After adding PFOA/PFNA, the $\alpha$-helix content of WT hL-FABP, S39G, M74G, and N111D variants increased with PFOA concentration, while the $\beta$-sheet content decreased slightly. The $\alpha$-helix and $\beta$-sheet contents in the $\mathrm{R} 122 \mathrm{G}$ variant showed no substantial change (Fig. 1; Table 1). The degree of CD spectral change was PFNA $>$ PFOA $>$ PFHxS/PFHxA. Except for the R122G variant, the content of $\alpha$-helices for
WT, S39G, M74G, and N111D variants increased with PFAA chain length, while $\beta$-sheet decreased.

Binding affinity of PFAAs for hL-FABP by fluorescence displacement assays

To obtain binding parameters of PFAAs for hL-FABP, the effect of titrating $0.25 \mu \mathrm{M}$ hL-FABP with increasing 
Table 1 Effect of PFAAs on the relative proportion of the secondary structure components of WT hL-FABP and its four variants as determined by $\mathrm{CD}$

\begin{tabular}{|c|c|c|c|c|c|c|c|c|c|c|}
\hline & \multicolumn{2}{|l|}{ WT } & \multicolumn{2}{|l|}{ S39G } & \multicolumn{2}{|l|}{$\mathrm{M} 74 \mathrm{G}$} & \multicolumn{2}{|l|}{ N111D } & \multicolumn{2}{|l|}{$\mathrm{R} 122 \mathrm{G}$} \\
\hline & $\begin{array}{l}\alpha \text {-Helices } \\
(\%)\end{array}$ & $\begin{array}{l}\beta \text {-Sheet } \\
(\%)\end{array}$ & $\begin{array}{l}\alpha \text {-Helices } \\
(\%)\end{array}$ & $\begin{array}{l}\beta \text {-Sheet } \\
(\%)\end{array}$ & $\begin{array}{l}\alpha \text {-Helices } \\
(\%)\end{array}$ & $\begin{array}{l}\beta \text {-Sheet } \\
(\%)\end{array}$ & $\begin{array}{l}\alpha \text {-Helices } \\
(\%)\end{array}$ & $\begin{array}{l}\beta \text {-Sheet } \\
(\%)\end{array}$ & $\begin{array}{l}\alpha \text {-Helices } \\
(\%)\end{array}$ & $\beta$-Sheet $(\%)$ \\
\hline No PFAAs & 17.1 & 50.3 & 16.9 & 50.4 & 16.6 & 51.2 & 17.5 & 49.1 & 15.3 & 51.5 \\
\hline PFHxS & 17.4 & 49.8 & 17.2 & 49.0 & 16.9 & 50.6 & 17.6 & 49.1 & 15.5 & 50.7 \\
\hline PFHxA & 16.9 & 50.0 & 17.2 & 49.8 & 16.5 & 50.5 & 17.5 & 49.3 & 15.4 & 50.9 \\
\hline PFOA & 17.5 & 46.8 & 17.6 & 47.4 & 17.1 & 48.7 & 17.9 & 48.4 & 15.1 & 49.6 \\
\hline PFNA & 17.8 & 47.9 & 18.0 & 48.6 & 17.4 & 48.6 & 18.3 & 47.4 & 14.9 & 50.8 \\
\hline
\end{tabular}

All data were determined by adding $100 \mu \mathrm{M}$ PFAAs to $5 \mu \mathrm{M}$ hL-FABP in $20 \mathrm{mM} \mathrm{NaH} \mathrm{PO}_{4} / \mathrm{Na}_{2} \mathrm{HPO}_{4}$ (pH 7.4)

concentrations of ANS was examined (Fig. S2A). The fluorescence intensity at $470 \mathrm{~nm}$, corrected for background (ligand only), was plotted as a function of total ligand concentration (Fig. 2a). Nonlinear regression analysis (Hill plot) of the binding data demonstrated that ANS bound to WT hL-FABP and S39G and M74G with an apparent dissociation constant $\left(K_{\mathrm{d}}\right)$, respectively (Fig. S2A). These results suggest a stoichiometry of one ANS molecule per hL-FABP binding site in the complex $(n=1.01-1.19$, Table 2). For N111D and R122G variants, however, no binding was detected (Table 2).

After the dissociation constant of ANS with hL-FABP was obtained, it was used as a fluorescence probe in the ligand displacement assays to measure the binding affinities of PFAAs with the protein (Figs. 2 and S2). The apparent inhibition constants or $K_{\mathrm{d}}$ derived from these experiments are documented in Table 2. The binding affinity of WT hL-FABP and its variants for PFHxA and PFHxS was not detected. The calculated $K_{\mathrm{d}}$ of PFOA and PFNA for S39G and M74G variants was similar to the wild type. However, binding affinities of PFOA and PFNA for N111D and R122G were not observed. Based on the fluorescence displacement assay, it was concluded that PFOA/PFNA displayed a moderate affinity for hL-FABP and decreased in the order PFNA $>$ PFOA $\gg$ PFHxS/PFHxA. These data indicate that hL-FABP was capable of binding structurally diverse nonfatty acid lipophilic environmental chemicals.

Thermodynamic analysis of the interaction of hL-FABP with PFAAs using ITC

To determine accurate $K_{\mathrm{d}}$ values of hL-FABP and PFAAs with different chain lengths, we performed ITC measurement (concentrations of hL-FABPs and PFAAs for ITC assay are given in Table S2). We did not observe binding affinity of WT hL-FABP and its variants for PFHxA. However, the binding affinities of WT hL-FABP and its variants for PFHxS were weak, with $\mathrm{K}_{\mathrm{d}}$ values ranging from 26.95 to $61.35 \mu \mathrm{M}$ for the variants (Table S3). The ITC measurements revealed that PFOA and PFNA could bind to WT hL-FABP binding sites at a 1:1 molar ratio, giving $K_{\mathrm{d}}$ values of 6.49 and $3.14 \mu \mathrm{M}$ (Table 3; Fig. 3), respectively, consistent with the ligand displacement assay results. Moreover, the $\Delta H$ for PFOA binding with WT hL-FABP was $-29.49 \mathrm{~kJ} / \mathrm{mol}$, much larger than $\Delta S(0.4 \mathrm{~J} / \mathrm{mol} / \mathrm{deg})$, indicating that the interaction was mainly mediated by electrostatic attraction and hydrogen bonding. The $K_{\mathrm{d}}$ values of PFOA and PFNA for S39G and M74G variants were similar to that of wild type. However, the binding affinities of N111D for PFOA and PFNA were lost. Interestingly, for R122G, the binding affinities for PFOA and PFNA were a bit lower than its wild type, while the molar ratio for PFOA/PFNA to the protein binding site changed to 1:2. Based on the ITC assay, it was concluded that PFOA/PFNA displayed a moderate affinity for hL-FABP and decreased in the order PFNA $>$ PFOA $>$ PFHxS $>$ PFHxA.

\section{Binding to PFOA protects hL-FABP from proteolysis}

Figure 4 shows the proteolytic peptide pattern that evolved from the digestion of hL-FABP with trypsin (Arg-C; Lys$\mathrm{C})$ in the apo- and holo-PFOA bound forms. The apo-form was significantly more susceptible to proteolysis than the holo-PFOA form. This indicated that PFOA binding stabilized the WT hL-FABP, S39G, and M74G structure. However, binding to PFOA protected N111D from proteolysis to some extent, but PFOA binding had no protection for R122G.

\section{Molecular simulations}

To gain insight into the role of the key residues, molecular docking coupled with molecular dynamics was performed to identify the interaction between PFAAs and the binding sites of L-FABP. PFAAs bound with two WT hL-FABP binding sites (Fig. 5a). In the outer binding site, PFAA entered in a heads-in orientation. Its head carboxyl made polar contact with the positive amide of Asn111 and 

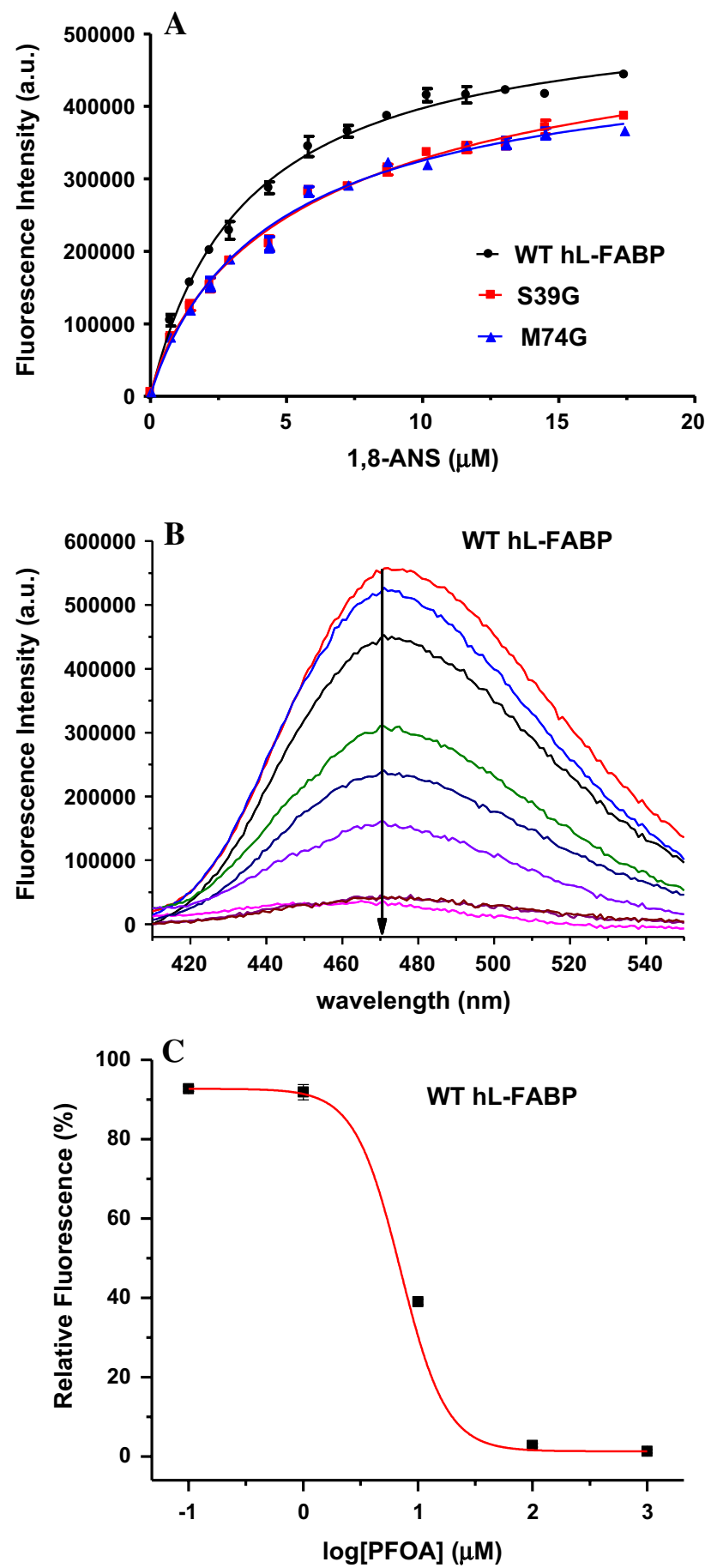

Fig. 2 Binding of fluorescence probe with hL-FABP. a Maximal fluorescence emission plot (measured at $470 \mathrm{~nm}$ ) for 1,8-ANS in the presence of $250 \mathrm{nM}$ of hL-FABP as a function of 1,8-ANS concentration. Values are mean $\pm \mathrm{SE}, n=3$. b Effect of increasing concentrations of PFOA on 1,8-ANS fluorescence. Fluorescence emission spectra of 1,8-ANS-hL-FABP in the presence of increasing concentrations of PFOA. Arrow indicates the direction of increasing PFOA concentration. c Relative fluorescence intensity of $12.25 \mu \mathrm{M} 1,8$ ANS in $250 \mathrm{nM} \mathrm{hL-FABP}$ as a function of added PFOA concentrations. Values are mean $\pm \mathrm{SE}, n=3$ the $\zeta \mathrm{NH}$ of Arg122 at the interface of the two binding sites. The PFAAs carboxyl was also involved in hydrogen bond interactions with three trapped water molecules in the binding cavity $\left(\mathrm{H}_{2} \mathrm{O} 353,243\right.$ and 227). The PFOA alkyl tail was stabilized by hL-FABP hydrophobic residues (Phe15, Ala54, Ile52, and Met113) (Fig. S4A). In the inner binding site, the PFAA head carboxyl made ionic contacts with the side chain of Ser39 and Arg122. The PFOA carboxyl was also involved in hydrogen bond interactions with the same three waters. The PFOA alkyl tail was accommodated in a hydrophobic cavity lined by Phe50, Ile52, and Thr102 (Fig. $\mathrm{S} 4 \mathrm{~B})$. The orientation of PFOA in the inner site was stabilized by the above hydrophobic contacts.

For the N111D variant, the substitution of Asn111 with Asp did not cause significant conformational change in the inner cavity comparison with WT hL-FABP. However, substitution of Asn111 with Asp disrupted the attraction of the positive amide of Asn111 to the negative carboxyl group of PFOA (Fig. 5b). It caused the dramatic reduction in the orientation potential for PFOA-hL-FABP interaction. In addition, the interaction between the PFOA anion head and the $\zeta \mathrm{NH}$ of Arg122 alone could not stabilize the heads-in mode. Consequently, it was difficult for PFOA to access the inner binding site due to the exclusion of the outer cavity (Fig. 5e).

Unlike the N111D variant, the substitution of Arg122 with glycine increased the volume of the outer binding pocket by $34 \%$, making it impossible for the outer binding site to form intensive hydrophobic stacking and hydrogen bonds with PFOA (Fig. 5c). However, the hydrogen bond between the carboxyl of PFOA and positive amide of Asn111 was retained to introduce PFOA in a headsin mode. For the R122G variant, PFOA still entered and bound to the inner binding site. Since Ser39 established a hydrogen bond network with the help of the trapped water molecules, PFOA was stabilized in the inner cavity (Fig. 5f).

The substitution of S39 with glycine in the variant did not have a large impact on the interaction between PFOA and hL-FABP (Fig. 5d, g). Without the hydrogen bonds between PFOA and Ser39 in the inner binding site, the charged anion group of PFOA established a hydrogen bond with $\operatorname{Arg} 122$, which stabilized PFOA. For M74G, the situation was similar to that of S39G (data not shown).

\section{Discussion}

Human liver cells are challenged by various lipophilic environmental xenobiotics, including PFAAs, which can be toxic at high concentrations. These xenobiotics can interact with some hepatic and transport proteins and disrupt 
Table 2 Binding parameters of PFOA and PFNA for WT $\mathrm{hL}-\mathrm{FABP}$ and its four variants determined fluorometrically by displacement of 1,8-ANS

$N B$ no binding detected

\begin{tabular}{|c|c|c|c|c|c|c|c|}
\hline & \multicolumn{3}{|c|}{ 1,8-ANS } & \multicolumn{2}{|l|}{ PFOA } & \multicolumn{2}{|l|}{ PFNA } \\
\hline & $\mathrm{R}^{2}$ & $K_{\mathrm{d}}(\mu \mathrm{M})$ & $n$ & $\mathrm{IC}_{50}(\mu \mathrm{M})$ & $K_{\mathrm{d}}(\mu \mathrm{M})$ & $\mathrm{IC}_{50}(\mu \mathrm{M})$ & $K_{\mathrm{d}}(\mu \mathrm{M})$ \\
\hline Wild type & 0.95 & $3.56 \pm 0.48$ & $1.01 \pm 0.08$ & $8.14 \pm 1.17$ & $2.36 \pm 0.34$ & $4.55 \pm 0.31$ & $1.32 \pm 0.20$ \\
\hline S39G & 0.96 & $6.64 \pm 0.96$ & $1.19 \pm 0.07$ & $7.16 \pm 0.46$ & $3.80 \pm 0.18$ & $4.06 \pm 0.53$ & $2.20 \pm 0.26$ \\
\hline $\mathrm{M} 74 \mathrm{G}$ & 0.95 & $4.79 \pm 0.79$ & $1.07 \pm 0.08$ & $6.68 \pm 0.92$ & $2.62 \pm 0.36$ & $4.93 \pm 0.45$ & $1.84 \pm 0.26$ \\
\hline N111D & $\mathrm{NB}^{\mathrm{a}}$ & $\mathrm{NB}^{\mathrm{a}}$ & $\mathrm{NB}^{\mathrm{a}}$ & $\mathrm{NB}^{\mathrm{a}}$ & $\mathrm{NB}^{\mathrm{a}}$ & $\mathrm{NB}^{\mathrm{a}}$ & $\mathrm{NB}^{\mathrm{a}}$ \\
\hline $\mathrm{R} 122 \mathrm{G}$ & $\mathrm{NB}^{\mathrm{a}}$ & $\mathrm{NB}^{\mathrm{a}}$ & $\mathrm{NB}^{\mathrm{a}}$ & $\mathrm{NB}^{\mathrm{a}}$ & $\mathrm{NB}^{\mathrm{a}}$ & $\mathrm{NB}^{\mathrm{a}}$ & $\mathrm{NB}^{\mathrm{a}}$ \\
\hline
\end{tabular}

Table 3 Thermodynamic parameters for the binding of PFOA to WT hL-FABP and its four variants as determined by ITC at $25^{\circ} \mathrm{C}$

\begin{tabular}{|c|c|c|c|c|c|c|c|}
\hline & & $N$ (sites) & $K_{\mathrm{a}}\left(\mathrm{M}^{-1}\right)$ & $K_{\mathrm{d}}(\mu \mathrm{M})$ & $\Delta H(\mathrm{~kJ} / \mathrm{mol})$ & $\Delta S(\mathrm{~J} / \mathrm{mol} / \mathrm{deg})$ & $\Delta G(\mathrm{~kJ} / \mathrm{mol})$ \\
\hline \multirow[t]{5}{*}{ PFOA } & WT & $0.999 \pm 0.016$ & $(1.54 \pm 0.14) \times 10^{5}$ & 6.49 & $-29.49 \pm 0.68$ & 0.4 & $-29.50 \pm 0.672$ \\
\hline & S39G & $1.08 \pm 0.024$ & $(1.21 \pm 0.13) \times 10^{5}$ & 8.26 & $-24.47 \pm 0.72$ & 15 & $-17.67 \pm 0.35$ \\
\hline & M74G & $0.984 \pm 0.013$ & $(1.12 \pm 0.09) \times 10^{5}$ & 8.93 & $-5.57 \pm 0.11$ & 4.0 & $-5.675 \pm 0.006$ \\
\hline & N111D & $\mathrm{ND}^{\mathrm{a}}$ & $(3.52 \pm 1.89) \times 10^{3}$ & 284.09 & $\mathrm{ND}^{\mathrm{a}}$ & $\mathrm{ND}^{\mathrm{a}}$ & $\mathrm{ND}^{\mathrm{a}}$ \\
\hline & $\mathrm{R} 122 \mathrm{G}$ & $0.554 \pm 0.042$ & $(1.19 \pm 0.32) \times 10^{5}$ & 8.40 & $-17.30 \pm 2.71$ & 27 & $-17.97 \pm 2.04$ \\
\hline \multirow[t]{5}{*}{ PFNA } & WT & $1.20 \pm 0.016$ & $(3.18 \pm 0.35) \times 10^{5}$ & 3.14 & $-5.07 \pm 0.09$ & 8.2 & $-5.275 \pm 0.113$ \\
\hline & S39G & $1.08 \pm 0.012$ & $(3.00 \pm 0.26) \times 10^{5}$ & 3.33 & $-5.10 \pm 0.08$ & 7.9 & $-5.301 \pm 0.122$ \\
\hline & M74G & $1.09 \pm 0.026$ & $(1.08 \pm 0.19) \times 10^{5}$ & 9.26 & $-5.39 \pm 0.20$ & 5.0 & $-5.513 \pm 0.073$ \\
\hline & N111D & $\mathrm{ND}^{\mathrm{a}}$ & $(8.84 \pm 1.77) \times 10^{3}$ & 113.12 & $\mathrm{ND}^{\mathrm{a}}$ & $\mathrm{ND}^{\mathrm{a}}$ & $\mathrm{ND}^{\mathrm{a}}$ \\
\hline & $\mathrm{R} 122 \mathrm{G}$ & $0.562 \pm 0.010$ & $(1.55 \pm 0.16) \times 10^{5}$ & 6.45 & $-8.49 \pm 0.21$ & -4.7 & $-8.609 \pm 0.086$ \\
\hline
\end{tabular}

${ }^{\text {a }} N D$ not detected

the biological function of liver cells. PFAAs have a similar structure to endogenous fatty acids, except that the hydrogen atoms of the fatty acids are replaced by fluorine atoms. Therefore, PFAAs may disrupt the binding of fatty acids to their transporters and finally disturb lipid regulation. In this study, PFAAs were validated as binding ligands by ITC binding assays, and PFOA and PFNA displayed a moderate affinity for hL-FABP.

Our simulation studies showed that PFOA and PFNA may bind with hL-FABP by a similar way of long-chain fatty acids binding with the protein. Sharma and Sharma (2011) revealed that hL-FABP had two binding sites with heads-in modes of entry for long-chain fatty acids, with the hL-FABP complex structure displaying an overall fold consisting of a $\beta$ barrel formed by ten antiparallel $\beta$ sheets and two $\alpha$ helices closing one end of the barrel. Sharma and Sharma (2011) further proposed that the encounter of the first fatty acid with Arg122 in hL-FABP triggered a conformational change in this residue, which then shunted the hydrophobic cargo to the inner fatty acid binding site in hL-FABP. Once the inner binding site was occupied, the outer one was free to receive the second fatty acid cargo, which again bound in a heads-in orientation stabilized by interactions with Asn111 and Arg122.

In this study, the ITC assay showed that PFOA bound with the R122G variant binding site at a molar ratio 1:2 was significantly different from WT hL-FABP and the other three variants. Thus, for $\mathrm{R} 122 \mathrm{G}$, we inferred that only one of two sites could interact with PFOA. Molecular simulation confirmed that the R122G variant engulfed PFOA only in the inner binding cavity, highlighting the crucial role of Arg 122 for PFAAs binding. The substitution of $\operatorname{Arg} 122$ with glycine increased the volume of the outer binding pocket, and this conformational change resulted in the inability of PFOA to bind with the outer binding site. Our CD results showed that the helical content for $\mathrm{R} 122 \mathrm{G}$ decreased, indicating that secondary structures changed and affected the outer binding site of R122G because two $\alpha$ helices were close to one end of the barrel and near the outer binding cavity (Sharma and Sharma 2011). This loosened or extended conformation was also supported by the limited proteolysis of $\mathrm{R} 122 \mathrm{G}$, which showed that it was significantly more susceptible to proteolysis than the wild type or the other holo-variant-PFOA complexes.

Molecular simulations revealed that the substitution of Asn111 with Asp did not cause obvious conformational change compared with the WT hL-FABP. Our CD experiment and the limited proteolysis of N111D supported the molecular simulation results. However, the substitution of Asn111 with Asp caused the dramatic reduction in the orientation potential of PFOA-L-FABP interaction. The interaction between the PFOA anion and the $\zeta \mathrm{NH}$ of $\operatorname{Arg} 122$ alone could not stabilize the heads-in mode, and the hydrophobic contact between PFOA and the outer binding site 

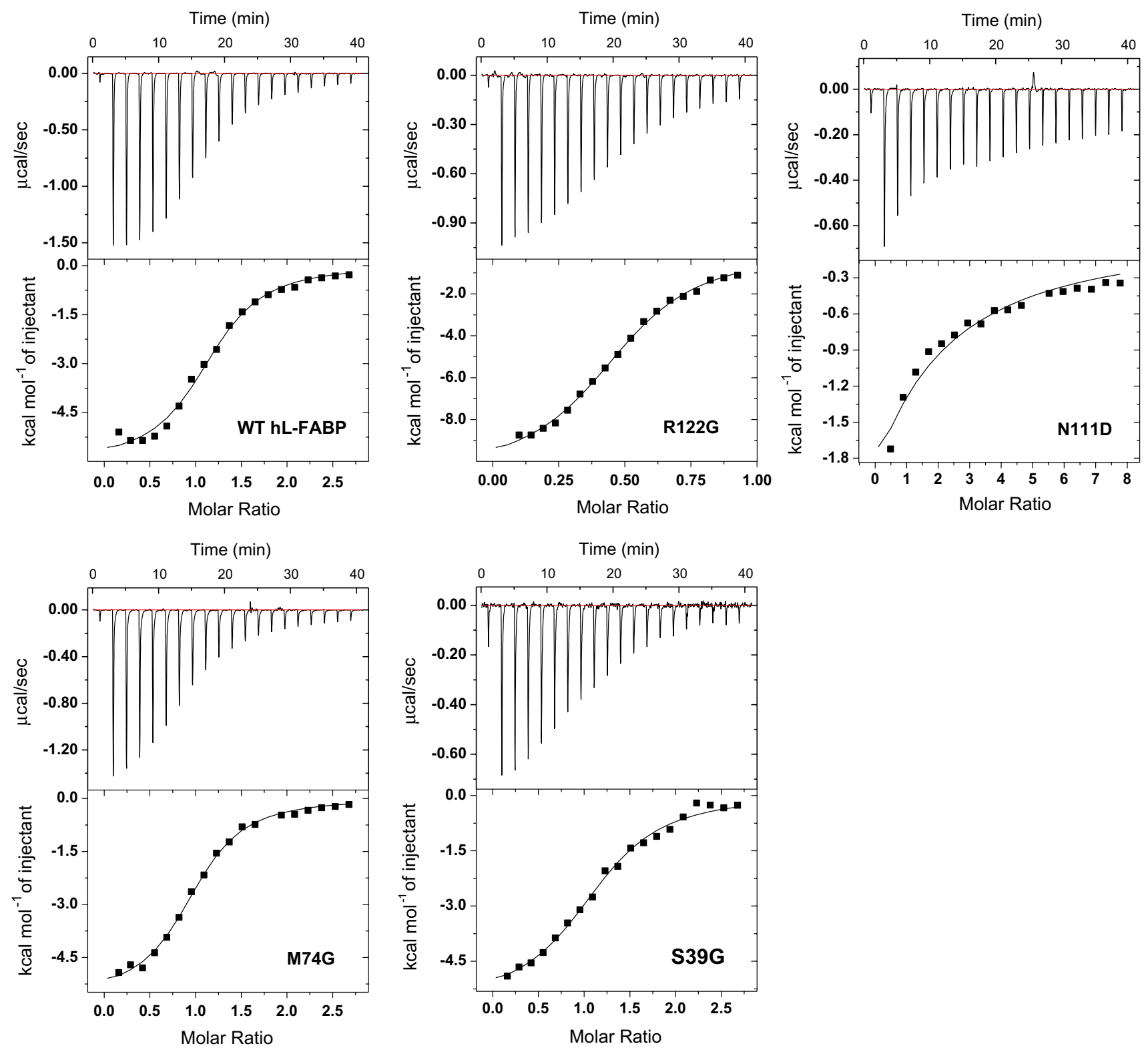

Fig. 3 ITC profiles for the binding of PFOA to WT hL-FABP and its four variants (S39G, M74G, N111D, and R122G) at $25^{\circ} \mathrm{C}$

was rather weak in comparison with that of its native fatty acid (octanoic acid), indicating that no binding to WT hLFABP was observed using the ITC assay (Fig. S5). Moreover, the electronegative nature of fluorine also results in the decrease in hydrophobic contact (Krafft and Riess 2009). Consequently, PFOA loses its access to the inner binding sites due to the exclusion of the outer cavity. The docking results were consistent with the ITC assay, which showed that the binding affinities of N111D for PFOA and PFNA were almost lost. This indicated that Asn111 plays a pivotal role in the heads-in mode of PFOA.

Taken together, our study demonstrated that hL-FABP can bind two molecules of PFAAs. We proposed the following scenario for PFAAs uptake by hL-FABP. The carboxyl head of the first PFOA with a heads-in orientation made polar contact with the positive amide of Asn111, and the $\zeta \mathrm{NH}$ of $\operatorname{Arg} 122$. Arg122 located at the interface of the two binding sites worked like a lever and pulled the first PFAAs inside. Because PFAAs only entered in a heads-in orientation, the opposite resulted in a direct clash of PFAA hydrophobic tails with $\operatorname{Arg} 122$. In the inner binding pocket, the PFAA head group made ionic contact with side chains of Arg122 and Ser39, and the alkyl tail was stabilized by hL-FABP hydrophobic residues.

PFAAs $(n>8)$ can activate PPAR activators (PPAR $\alpha$ and $\operatorname{PPAR} \gamma$ ), as well as the constitutive androstane 
receptor and pregnane $\mathrm{X}$ receptor (Ding et al. 2009; Fang et al. 2012; Vanden Heuvel et al. 2006). As these nuclear hormone receptors are located in the nucleus of the

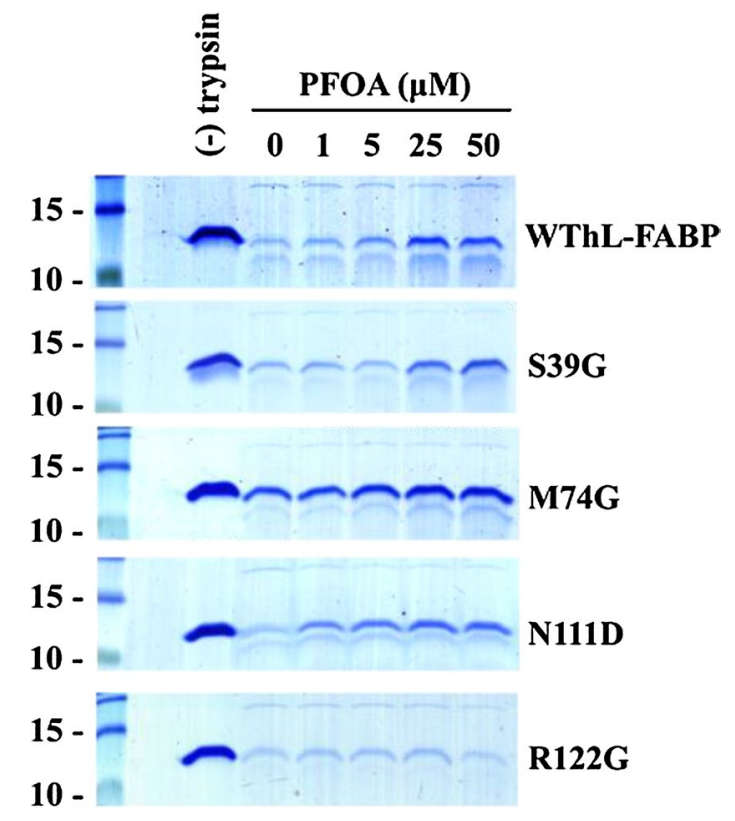

Fig. 4 Electrophoretic band of WT hL-FABP and its four variants (S39G, M74G, N111D, and R122G) digested with trypsin hepatocyte, transport of these relatively insoluble lipophilic environmental pollutants from the cytoplasm to the nucleus seems to be required for activation of the PPARs transcriptional response. Researchers have provided convincing evidence that hL-FABP directly interacts with PPAR $\alpha$ and is involved in the nucleo-cytoplasmic shuttling of activator ligands (Hostetler et al. 2009; Wolfrum et al. 2001). Thus, we speculated that L-FABP may act as a carrier to deliver PFAAs to PPARs and then disrupt PPAR pathways.

Finally, based on the binding affinity of PFAAs for hLFABP obtained in this study, we estimated the concentration of PFAAs bound to hL-FABP as follows:

$[\mathrm{PFAA}-\mathrm{P}]+[\mathrm{PFAA}]=[\mathrm{PFAA}]_{\text {total }}$

$[\mathrm{PFAA}-\mathrm{P}]+[\mathrm{P}]=[\mathrm{P}]_{\text {total }}$

$[\mathrm{PFAA}-\mathrm{P}]=K_{\mathrm{b}}[\mathrm{PFAA}][\mathrm{P}]$

In above equation, [PFAA-P] is the concentration of protein-bound PFAA, [PFAA] is the concentration of unbound PFAA, $[\mathrm{PFAA}]_{\text {total }}$ is the total concentration of PFAA, $[\mathrm{P}]$ is the concentration of free hL-FABP, $[\mathrm{P}]_{\text {total }}$ is the total concentration of hL-FABP, which in the physiological condition is $200-400 \mu \mathrm{M}$ in hepatocyte cytosol (Lawrence et al.

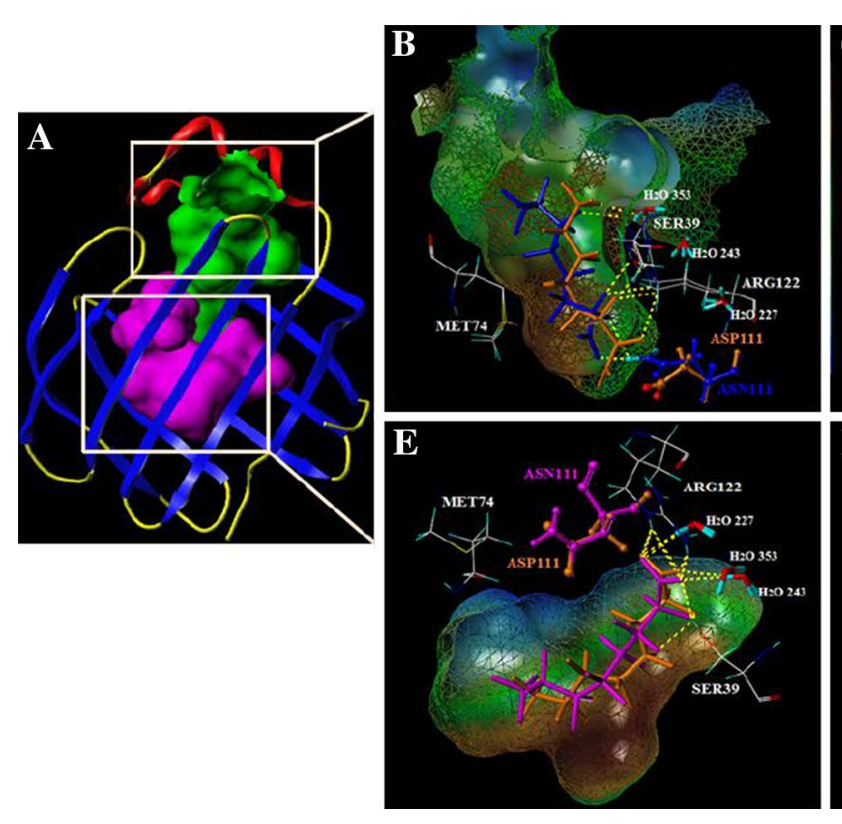

Fig. 5 Comparison between the wild type and residue substitution hL-FABP active pockets. a Whole structures of hL-FABP, outer binding site is indicated as a green pocket, and inner binding site is shown in magenta. b-d represent the outer pocket comparison between the wild type (opaque diagram) and the Arg122, Asn111, and Ser39 mutant proteins (gridding diagram), respectively. $\mathbf{e}-\mathbf{g}$ represent the inner pocket comparison between the wild type (opaque diagram) and the Arg122, Asn111, and Ser39 mutant proteins (gridding dia-
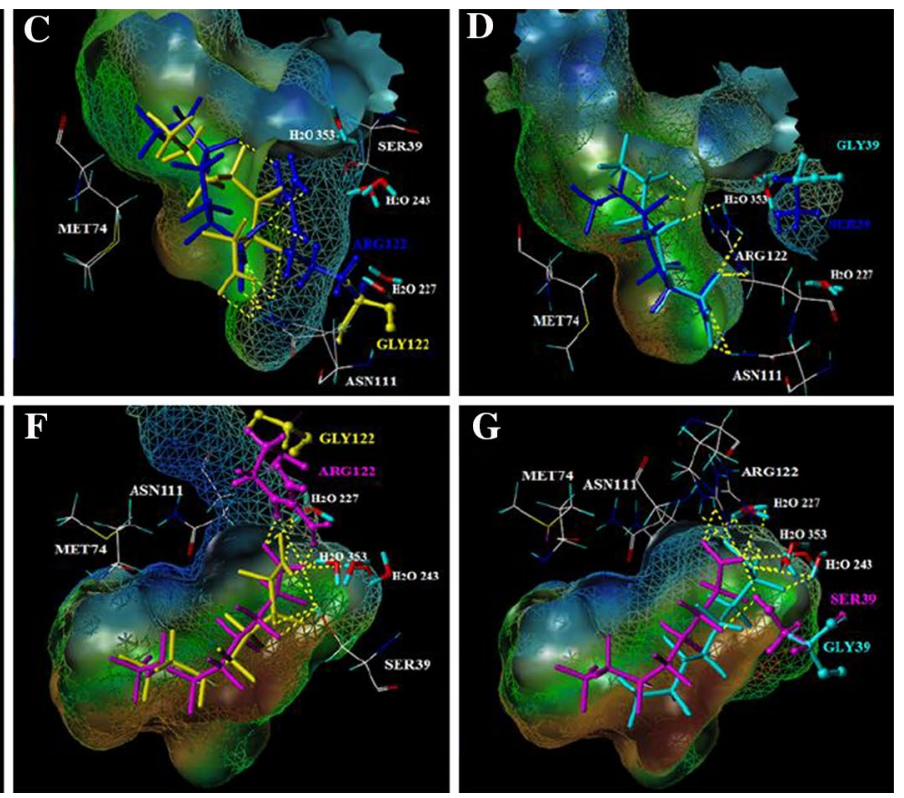

gram), respectively. The binding poses of PFOA in the outer and inner binding sites of the wild-type pockets are illustrated by blue and magenta sticks, respectively. The binding poses of PFOA in the wildtype pocket for Arg122, Asn111, and Ser39 mutation cavities are illustrated by yellow, orange, and cyan sticks, separately. The hydrogen bonds are shown by yellow dashed lines. Trapped water molecules are also shown (color figure online) 
$2000)$, and $K_{\mathrm{b}}$ is the equilibrium association constant $\left(1 / K_{\mathrm{d}}\right)$ of PFAA with the protein.

The geometric mean concentration of PFOA in their serum is $2.2 \mu \mathrm{M}$ for occupational workers, while the concentration is only $12 \mathrm{nM}$ for the general human population (Olsen et al. 2007). According to the mean ratio of liver to blood given in the literature (Maestri et al. 2006), the liver concentration of PFOA in occupational workers is between 2.86 and $5.94 \mu \mathrm{M}$. The calculated concentration of hL-FABP-PFOA for the general population was $0.05 \mu \mathrm{M}$ and that for occupational workers was 2.77-5.85 $\mu \mathrm{M}$. Thus, the concentrations of hL-FABP bound by PFOA in both populations were insignificant. However, for workers with the highest concentration of PFOA $(275.6 \mu \mathrm{M})$ in their serum (Ehresman et al. 2007; Sakr et al. 2007), the concentration of hL-FABP-PFOA reached 186.43 $\mu \mathrm{M}$. This suggested as much as $46-93 \%$ of hL-FABP was bound by PFOA, and since hL-FABP is a transport protein, displacement would impair the uptake and transport of fatty acids. Taken together, more attention should be paid toward the potential toxic effects of PFAAs on humans, or at least occupational workers.

\section{Conclusions}

This is the first report on the accurate binding constant and role of specific sites in the binding interaction of hL-FABP with PFAAs. The binding affinity of PFAAs increased significantly with their carbon number. The driving forces for the binding of PFAAs with hL-FABP were predominantly hydrophobic and hydrogen-bonding interaction. Arg 122 and Asn111 played a pivotal role in these interactions. These findings may help understand the distribution pattern, bioaccumulation, elimination, and toxicity of PFAAs in humans.

Acknowledgments This work was supported by the Strategic Priority Research Program of the Chinese Academy of Sciences (XDB14000000) and the National Natural Science Foundation of China (grants 31320103915 and 31025006 for Dai, 21277164 for Zhang).

Conflict of interest The authors declare they have no competing financial interests.

\section{References}

Atshaves BP, Martin GG, Hostetler HA, McIntosh AL, Kier AB, Schroeder F (2010) Liver fatty acid-binding protein and obesity. J Nutr Biochem 21:1015-1032

Bernlohr DA, Simpson MA, Hertzel AV, Banaszak LJ (1997) Intracellular lipid-binding proteins and their genes. Annu Rev Nutr 17:277-303

Carbone V, Velkov T (2013) Interaction of phthalates and phenoxy acid herbicide environmental pollutants with intestinal intracellular lipid binding proteins. Chem Res Toxicol 26:1240-1250

De Silva AO, Mabury SA (2006) Isomer distribution of perfluorocarboxylates in human blood: potential correlation to source. Environ Sci Technol 40:2903-2909

Ding L, Hao F, Shi Z, Wang Y, Zhang H, Tang H et al (2009) Systems biological responses to chronic perfluorododecanoic acid exposure by integrated metabonomic and transcriptomic studies. J Proteome Res 8:2882-2891

Ehresman DJ, Froehlich JW, Olsen GW, Chang SC, Butenhoff JL (2007) Comparison of human whole blood, plasma, and serum matrices for the determination of perfluorooctanesulfonate (pfos), perfluorooctanoate (pfoa), and other fluorochemicals. Environ Res 103:176-184

Fang X, Zou S, Zhao Y, Cui R, Zhang W, Hu J et al (2012) Kupffer cells suppress perfluorononanoic acid-induced hepatic peroxisome proliferator-activated receptor alpha expression by releasing cytokines. Arch Toxicol 86:1515-1525

Hostetler HA, McIntosh AL, Atshaves BP, Storey SM, Payne HR, Kier AB et al (2009) L-fabp directly interacts with pparalpha in cultured primary hepatocytes. J Lipid Res 50:1663-1675

Kannan K, Corsolini S, Falandysz J, Fillmann G, Kumar KS, Loganathan BG et al (2004) Perfluorooctanesulfonate and related fluorochemicals in human blood from several countries. Environ Sci Technol 38:4489-4495

Kelly SM, Jess TJ, Price NC (2005) How to study proteins by circular dichroism. Biochim Biophys Acta 1751:119-139

Kennedy GL Jr, Butenhoff JL, Olsen GW, O’Connor JC, Seacat AM, Perkins RG et al (2004) The toxicology of perfluorooctanoate. Crit Rev Toxicol 34:351-384

Krafft MP, Riess JG (2009) Chemistry, physical chemistry, and uses of molecular fluorocarbon-hydrocarbon diblocks, triblocks, and related compounds-unique "apolar" components for self-assembled colloid and interface engineering. Chem Rev 109:1714-1792

Lau C, Anitole K, Hodes C, Lai D, Pfahles-Hutchens A, Seed J (2007) Perfluoroalkyl acids: a review of monitoring and toxicological findings. Toxicol Sci 99:366-394

Lawrence JW, Kroll DJ, Eacho PI (2000) Ligand-dependent interaction of hepatic fatty acid-binding protein with the nucleus. J Lipid Res 41:1390-1401

Luebker DJ, Hansen KJ, Bass NM, Butenhoff JL, Seacat AM (2002) Interactions of fluorochemicals with rat liver fatty acid-binding protein. Toxicology 176:175-185

Maestri L, Negri S, Ferrari M, Ghittori S, Fabris F, Danesino P et al (2006) Determination of perfluorooctanoic acid and perfluorooctanesulfonate in human tissues by liquid chromatography/single quadrupole mass spectrometry. Rapid Commun Mass Spectrom 20:2728-2734

Olsen GW, Burris JM, Ehresman DJ, Froehlich JW, Seacat AM, Butenhoff JL et al (2007) Half-life of serum elimination of perfluorooctanesulfonate, perfluorohexanesulfonate, and perfluorooctanoate in retired fluorochemical production workers. Environ Health Perspect 115:1298-1305

Renner R (2001) Growing concern over perfluorinated chemicals. Environ Sci Technol 35:154A-160A

Sakr CJ, Leonard RC, Kreckmann KH, Slade MD, Cullen MR (2007) Longitudinal study of serum lipids and liver enzymes in workers with occupational exposure to ammonium perfluorooctanoate. $\mathrm{J}$ Occup Environ Med 49:872-879

Sharma A, Sharma A (2011) Fatty acid induced remodeling within the human liver fatty acid-binding protein. J Biol Chem 286:31924-31928

Sreerama N, Woody RW (2000) Estimation of protein secondary structure from circular dichroism spectra: comparison of contin, selcon, and cdsstr methods with an expanded reference set. Anal Biochem 287:252-260 
Steenland K, Tinker S, Frisbee S, Ducatman A, Vaccarino V (2009) Association of perfluorooctanoic acid and perfluorooctane sulfonate with serum lipids among adults living near a chemical plant. Am J Epidemiol 170:1268-1278

Vanden Heuvel JP, Thompson JT, Frame SR, Gillies PJ (2006) Differential activation of nuclear receptors by perfluorinated fatty acid analogs and natural fatty acids: a comparison of human, mouse, and rat peroxisome proliferator-activated receptor-alpha, -beta, and -gamma, liver $\times$ receptor-beta, and retinoid $\times$ receptoralpha. Toxicol Sci 92:476-489

Velkov T, Chuang S, Wielens J, Sakellaris H, Charman WN, Porter CJ et al (2005) The interaction of lipophilic drugs with intestinal fatty acid-binding protein. J Biol Chem 280:17769-17776
Weiss J, Andersson P, Lamoree M, Leonards P, van Leeuwen S, Hamers T (2009) Competitive binding of poly- and perfluorinated compounds to the thyroid hormone transport protein transthyretin. Toxicol Sci 109:206-216

Wolfrum C, Borrmann CM, Borchers T, Spener F (2001) Fatty acids and hypolipidemic drugs regulate peroxisome proliferator-activated receptors alpha- and gamma-mediated gene expression via liver fatty acid binding protein: a signaling path to the nucleus. Proc Natl Acad Sci U S A 98:2323-2328

Zhang W, Zhang Y, Taniyasu S, Yeung LW, Lam PK, Wang J et al (2013) Distribution and fate of perfluoroalkyl substances in municipal wastewater treatment plants in economically developed areas of china. Environ Pollut 176:10-17 